\title{
An Uncertainty Model of the Professional Partnership
}

\author{
by Gabriel A. Hawawini*
}

\section{Introduction}

Consider a professional partnership. Payments for the services performed by partners are usually either on a contingency fee basis or on a noncontingency fee basis. Under contingency fee contracts (CFC hereafter), compensation for services rendered is payable only if the partners have sucessfully completed the task they were hired to perform. For example, with this method, payment to lawyers would be a percentage of the recovery or benefits awarded the client as a result of the service performed by the lawyers. Under noncontingency fee contracts (NCFC hereafter), compensation for the services rendered is paid to partners regardless of the outcome of the service performed.

The purpose of this paper then is to develop a stochastic model of the professional partnership that incorporates both a random income from CFC operations as well as the attitudes of the partners toward risk, and to examine the effect of uncertainty on the behavior of the partnership and its clients.

It is shown that most of the conclusions reached in the case of the partnership operating in a world of certainty are modified by the presence of uncertain CFC operations. Moreover, the policy implications that can be inferred from the certainty model are somewhat weakened when a stochastic model of the partnership is considered. For instance, the equilibrium employment and output levels are different from those attained by the partnership operating in a world of certainty and the possibility of a conflict between partners and their clients that may arise from different equilibrium solutions for the two groups is shown to be considerably smaller under uncertainty when partners and their clients are risk averse individuals.

The remaining part of this paper is organized as follows. In the next section the assumptions of a stochastic model of the professional partnership are stated and discussed. For the purpose of exposition, the law firm is taken as an example of a professional partnership. The third section examines the case of the firm that performs services only under CFC, and investigates the impact of risk on the equilibrium position of the firm and its clients. The model is then subjected to a comparative-statics analysis in order to determine the behavior of the

* Professor of Finance, INSEAD, Fontainebleau, France. An earlier version of this paper was presented at the Tenth Seminar of The European Group of Risk and Insurance Economists in Rome (Italy), September 1983. Useful comments were made by Professor Cheng-few Lee and an anonymous referee. 
firm in response to changes in the parameters of the model. The fourth section analyzes an extended model of the firm that incorporates both CFC operations (the risky activity) and NCFC operations (the riskless activity). The last section contains a discussion on the policy implications of the models and some concluding remarks.

\section{Assumptions and notations of the model}

\subsection{Production}

It is assumed that the firm's partners and their clients contractually agree on the relative share $b(0<b<1)$ of the "output" to be split between them. The traditional concept of output is given a different interpretation in this context. It is defined as the monetary value of the benefits received by the clients as a result of the services performed by the partners. This homogeneous output is measured in dollars and assumed to be a function of two variable inputs and two fixed inputs. The two variable inputs are the number of partners $(N)$ and the number of non-partner personnel $(n)$ (such as, for example, paralegals and other supporting staff working in law firms). The two fixed inputs are capital $(K)$ such as office space and information systems (the legal library is an example) and the "case loads" $(C)$ from which the partners can draw.

To illustrate, consider the case of a law firm. Here, the "case loads" $(C)$ can be viewed as the total monetary value of all cases under litigation, namely, the "inventory" of all available cases. Assume the law firm has a production function which displays diminishing marginal product for both lawyer and non-lawyer inputs and which is well behaved, continuous with continuous first- and second-order partial derivatives.

$$
Q=Q(N, n, C, K)
$$

$$
\text { with } Q_{N}, Q_{n}>0
$$

$$
\begin{aligned}
& Q_{N N}, Q_{n n}<0 \\
& Q_{N N} Q_{n n}-Q_{N n}^{2}>0 \\
& Q_{N n}=Q_{n N}>0
\end{aligned}
$$

where subscripts indicate the order and the variable with respect to which the partial derivative is computed.

The extended model developed in section 4 differenciates between two products. Those produced under CFC which are risky and those produced under NCFC which are riskless. Production under CFC will be identified by the subscript one and production under NCFC by the subscript two. The risky output produced under CFC is a random variable $Q_{1}^{*}$ defined as

$$
Q_{1}^{*}=Q_{1}\left(N_{1}, n_{1} ; \bar{C}, K\right) u
$$

where $u$ is a positive multiplicative random variable with a density function $G(u)$ which summarizes the beliefs of the partners as to the probable outcomes for the monetary value of the recovery or benefits awarded to the firm's clients. The random variable $u$ is further assumed to 
be unrelated to the number of partners $N$, that is, $d u / d N=0$. Under condition of certainty the random variable $u$ takes the value of one and $Q_{1}^{*}$ equals $Q_{1}$. Under uncertainty, the output $Q_{1}^{*}$ must be reinterpreted to represent the ex ante production given an expected case loads $\bar{C}$ instead of its certainty value $C$.

\subsection{Lawyers' and clients' attitudes toward risk}

The model developed in this paper incorporates the attitudes of lawyers and their clients toward the risk associated with legal cases whose outcome is uncertain. It is assumed that attitudes toward risk can be summarized in a von Neumann-Morgenstern [15] utility function of wealth $U(\cdot)$ characterized by a positive and decreasing marginal utility of wealth, that is, $U^{\prime}(\cdot)>0$ and $U^{\prime \prime}(\cdot)<0$, conditions that imply risk aversion. Under risk neutrality $U^{\prime}(\cdot)>0$ and $U^{\prime \prime}(\cdot)=0$. It is implicitly assumed that group preferences satisfy the transitivity axiom and thus the utility function adequately represents group preferences.

It is a standard procedure in stochastic optimization models in economics - which is supported by casual empiricism - to also assume that individuals exhibit nonincreasing absolute risk aversion. ' That is, their aversion to risk remains constant or, more likely, declines as their wealth increases.

\section{The model with all cases under contingency fee contracts}

\subsection{Firm's objective function and equilibrium position}

In this section the examination is restricted to the case where the partnership operates exclusively under CFC, its risky output.

Consider first the simplest case where partners are the only input and thus $Q^{*}=Q(N) u$. The objective function of the partnership is to maximize $Z_{1}$, the expected utility of the random income per partner $Y_{1}^{2}$

$$
Z_{1}=E U\left(Y_{1}\right)=E U\left(\left(b Q^{*}-F\right) / N\right)
$$

where $F$ is the cost of the firm's fixed capital, $b$ is a parametrically given share proportion ${ }^{3}$, and

${ }^{1}$ Risk aversion alone does not completely describe an individual's attitude toward risk when the model is subjected to a comparative statics analysis as it is shown in the next two sections. Arrow [1] and Pratt [12] have demonstrated that an index of absolute risk aversion, defined as $R_{A}($.) $=$ $-U^{\prime \prime}(.) / U^{\prime}($.$) , must be introduced into the analysis in order to reach any meaningful comparative-stat-$ ics results. An individual is said to display increasing, constant or decreasing absolute risk aversion according to his index of absolute risk aversion $R_{A}($.$) increases, remains constant or decreases with$ wealth. The model that follows is essentially based on the Arrow-Pratt risk aversion assumption. It should be pointed out, however, that Kihlstrom et al. [7] have recently generalized Arrow-Pratt's results by allowing the existence of random initial wealth.

${ }^{2}$ This is similar to the approach used for the case of the cooperative firms. See Ward [16], Domar [3], Vanek [14], Meade [9] and Oi and Clayton [10]. Kwon [8] was the first to employ this framework to the modelling of the behaviour of the law firm under certainty.

${ }^{3}$ The share proportion $b$ is usually suggested by bar associations through minimum fee schedules. 
$E$ the expectation operator. Differenciating $Z_{1}$ with respect to $N$, the number of partners, gives the first order condition for an extremum

$$
d Z_{1} / d N=(1 / N) E U^{\prime}\left(Y_{1}\right)\left(b Q_{N}^{*}-Y_{1}\right)=0
$$

in which both $Q^{*}$ and $Y_{1}$ are random variables. Given risk aversion $\left(U^{\prime \prime}<0\right)$ and diminishing marginal product for partners, the firm maximizes the net income per partner since the second order condition is satisfied

$$
d^{2} Z_{1} / d_{N}^{2}=D_{1}=(1 / N)^{2} E U^{\prime \prime}\left(Y_{1}\right)\left(b Q_{N}-Y_{1}\right)^{2}+(1 / N) E U^{\prime}\left(Y_{1}\right) b Q_{N N}^{2}<0 .
$$

Notice that a finite and unique solution to the maximization problem exists even under constant marginal product when risk aversion is displayed.

Noting that $E(A B)=\operatorname{Cov}(A, B)+E(A) E(B)$, equation (4) can be rewritten as ${ }^{4}$

$$
\begin{aligned}
& E\left(b Q_{N}^{*}\right)=E Y_{1}-\lambda \operatorname{Cov}\left(U^{\prime}, u\right) / E U^{\prime} \\
& \text { with } \lambda=(b Q / N)(e-1) \text { and } e=Q_{N} /(Q / N) .
\end{aligned}
$$

The coefficient $e$ is the elasticity of the production function with respect to $N$. By definition, the covariance has the sign of the derivative $d U^{\prime} / d u$. This derivative is equal to $U^{\prime \prime} d Y_{1} / d u$ and hence is negative under risk aversion since $U^{\prime \prime}<0$ and $d Y_{1} / d u>0$. Because production takes place over the input range for which the marginal product is smaller than the average product, and since uncertainty is multiplicative, it follows that $e<1$ and thus $\lambda<0$. Therefore

$$
E\left(b Q_{N}^{*}\right)<E Y_{1}
$$

That is, in equilibrium, the mathematical expectation of the net value of the marginal product (VMP hereafter) of partners is smaller than the expected net income per partner. Under certainty $u=1$, the covariance is zero, and the expected values are equal to their argument. Consequently, the VMP of partners equals the net income per partner at the certainty equilibrium position. Referring to figure 1, observe the increase in the number of partners under uncertainty from the certainty level of employment $N_{1}$ to the uncertainty level $N_{2}$ prevailing when the firm is risk averse and satisfies inequality (7). Since there exists a one-to-one correspondance between the level of employment and the level of production, it follows that the volume of services performed by the risk averse partnership, operating under a benefit sharing arrangement with its clients, exceeds the certainty volume. Although this result may at first appear paradoxical, it is not so. Being risk averse, the partnership expands the membership of partners in order to reduce the variability of the net income of each member. ${ }^{5}$

\footnotetext{
${ }^{4}$ Note that $(b Q *-Y)=b(Q / N)(e-1) u+F / N$

and thus $\operatorname{Cov}\left(U^{\prime}, b Q_{N}^{*}-Y\right)=\lambda \operatorname{Cov}\left(U^{\prime}, u\right)$.

For the sake of compactness $U^{\prime}$ is short for $U^{\prime}(Y)$.

${ }^{5}$ Note that since $Y_{1}=\left(b Q^{*}-F\right) / N$ then $\sigma\left(Y_{1}\right)=b(Q / N) \sigma(u)$

and $d \sigma\left(Y_{1}\right) / d N=(\lambda / N) \sigma(u)<0$. An increase in employment $N$ reduces the variability of the net income per lawyer $\sigma\left(Y_{1}\right)$.
} 


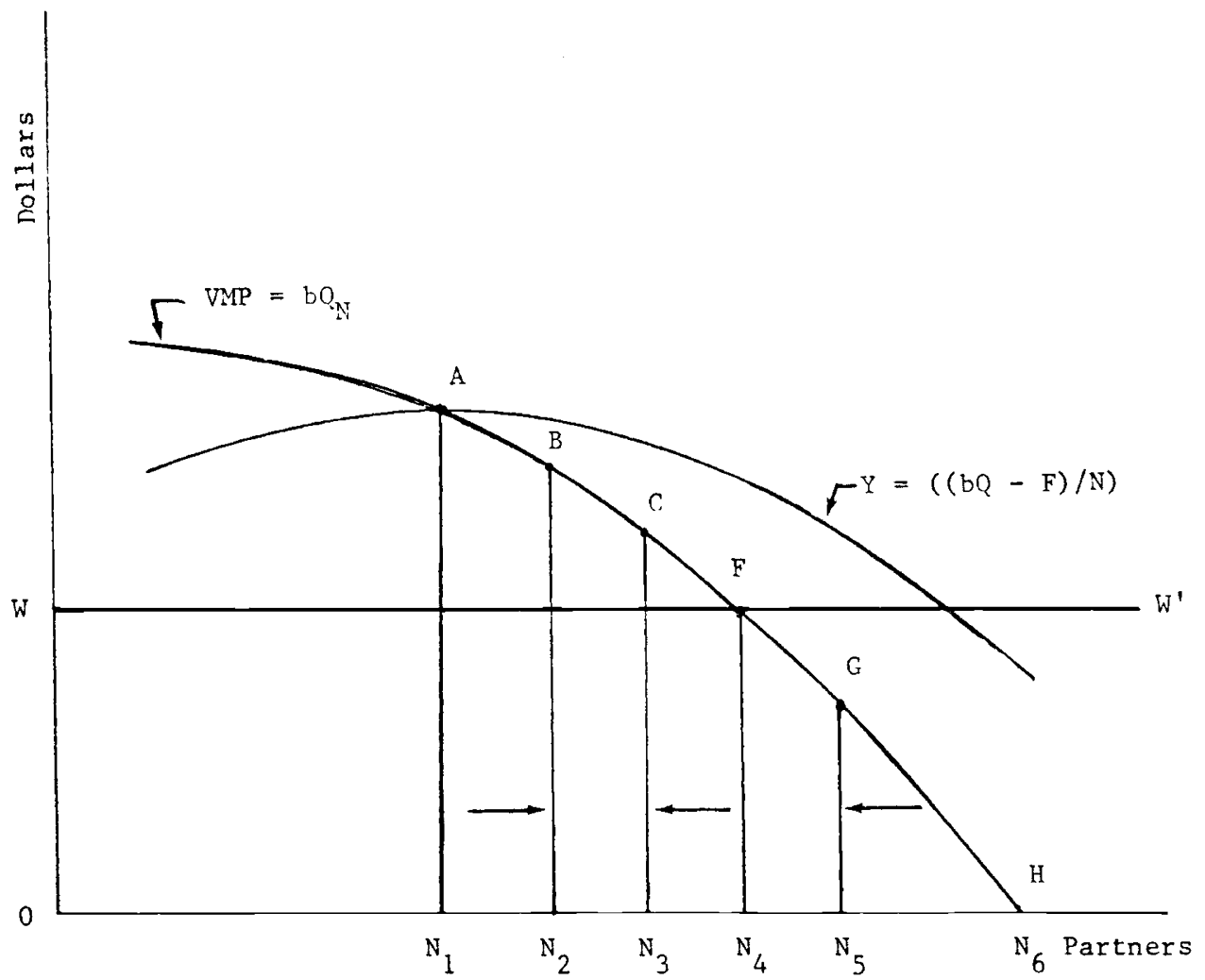

Figure I

A: Certainty Equilibrium, Per-Partner-Income Maximization.

B: Risk Aversion Equilibrium, Per-Partner-Income Maximization.

C: Risk Aversion Equilibrium, Profits Maximization.

F: Certainty Equilibrium, Profits Maximization.

G: Clients Equilibrium Under Risk Aversion.

H: Clients Equilibrium Under Certainty.

The case of the profit-maximizing partnership is worth examining and comparing to that of the partnership that maximizes the net income per partner. Suppose that partners can earn the competitive wage $W$ in the market place, then the maximization of the expected utility of profits yields the condition $E U^{\prime}\left(b Q_{N}^{*}-W\right)=0$ which can be rewritten as

$$
E\left(b Q_{N}^{*}\right)=W-\left(b Q_{N}\right) \operatorname{Cov}\left(U^{\prime}, u\right) / E U^{\prime}>W .
$$


That is, the risk averse partnership that maximizes the expected utility of profits will, in equilibrium, set the expected value of the VMP of partners above the opportunity competitive wage rate $W$ for partners. Under certainty the covariance is zero and the partnership will equate the VMP of partners to their prevailing competitive wage rate.

Referring to figure 1, observe that for the profit-maximizing partnership equilibrium is at point $\mathrm{F}$ where the opportunity wage line $W W^{\prime}$ intersects the VMP curve. To this point corresponds the employment of $N_{4}$ partners compared to $N_{1}$ partners for the per-member-incomemaximizing partnership. Under uncertainty and risk aversion the expected-utility-of-profitmaximizing partnership is in equilibrium at point $\mathrm{C}$ where $E(V M P)>W$. To this point corresponds the employment of $N_{3}$ partners. Thus the risk averse profit-maximizing partnership employs and produces less under uncertainty ${ }^{6}$ in opposition to the per-member-incomemaximizing partnership that employs and produces more under uncertainty and risk aversion at the equilibrium point $B$. Consequently, the employment and production gaps between the two types of firms is narrower under uncertainty and risk aversion than under certainty. The certainty gap measured by the distance $N_{1} N_{4}$ is reduced to an uncertainty gap measured by the distance $N_{2} N_{3}$. Uncertainty and risk aversion have the effect of weakening the difference between the per-partner-income-maximizing firm and the profit-maximizing firm. ${ }^{7}$

\subsection{Comparative-statics behavior}

Given that the partnership is in a position of equilibrium it is possible to investigate the effect on membership and output of a change in one of the firm's exogenous variables, namely, the share proportion $(b)$, the fixed costs on capital $(F)$ and the moments of the distribution of the random variable $u$. Comparative-static results are examined below and summarized in the first column of table 1 .

To determine the impact of a change in share proportion $b$, differentiate implicitly equation (4) with respect to $b$ and to $N$. It is shown in the mathematical appendix that

$$
d N / d b=-\left(1 / D_{1} N^{2}\right)\left\{(Q / \lambda)\left[E U^{\prime \prime}(\lambda u+F / N)^{2}-(F / N) E U^{\prime \prime}(\lambda u+F / N)\right]-(F / b) E U^{\prime}\right\}
$$

where both $D_{1}$ and $\lambda$ are negative. The first term in brackets is clearly negative under risk aversion. The sign of the second term depends on the type of absolute risk aversion displayed by partners. Following Sandmo [13] it can be easily shown that the sign of the second term is negative, zero or positive according as decreasing, constant or increasing absolute risk aversion is displayed. ${ }^{8}$ This important result will be used repeatedly in the rest of the paper. Finally, the last term in the RHS of equation (9) is always positive. Since $\lambda$ is negative, the sign [13].

${ }^{6}$ For the impact of price uncertainty on the theory of the competitive firm see Baron [2] and Sandmo

${ }^{7}$ See Paroush and Kahana [11], Hawawini and Michel [4] and Hawawini [5] for the effect of price uncertainty on the cooperative firm. For a comparative analysis of the cooperative and the competitive firms see Hawawini [6].

${ }^{8}$ Sandmo has shown that $E U^{\prime \prime}\left(p-C^{\prime}(x)\right) \lesseqgtr 0$ according as $R_{A}^{\prime} \lesseqgtr 0$ where $p$ is a positive random variable and $C^{\prime}(x)>0$.

Here we have $E U^{\prime \prime}(\lambda+F / N)=\lambda E U^{\prime \prime}(u+F /(\lambda N))$. Since $u$ is a positive random variable and $F /(\lambda N)<0$, Sandmo's results hold for $E U^{\prime \prime}(u+F /(\lambda / N))$ and are reversed for $E U^{\prime \prime}(\lambda u+F / N)$ since $\lambda<0$. 
of $d N / d b$ is indeterminate regardless of the type of risk aversion displayed by partners. The only case for which the sign of equation (9) can be determined is when the firm is risk neutral, in which case $U^{\prime \prime}=0$ and the derivative is negative. The derivative is also negative under certainty. Given risk aversion and diminishing marginal product for partners one cannot make a definite statement as to the response of the firm to changes in share proportion except that $d N / d b$ becomes negative as the firm's aversion to risk diminishes and approaches the limit case of risk neutrality. It is clear that this result weakens considerably the policy implication that can be drawn from the case of certainty or risk neutrality where $d N / d b$ is definitely negative and a change in the share proportion leads to a change in employment and output in the opposite direction. We will return to this point in the last section.

The response of the partnership to a change in fixed capital costs is obtained by differentiating implicity equation (4) with respect to $F$ and to $N$ which gives

$$
d N / d F=\left(1 / D_{1} N^{2}\right)\left\{E U^{\prime \prime}(\lambda u+F / N)-E U^{\prime}\right\}>0 .
$$

The first term in braces is nonpositive under nonincreasing absolute risk aversion and the second is positive in general. Consequently, nonincreasing absolute risk aversion is a sufficient but not necessary condition for the partnership operating under uncertain CFC to vary the number of partners and the volume of its legal services directly with its fixed capital costs. ${ }^{9}$ This result is similar to that of the partnership operating in a world of certainty except that under uncertainty the partnership should display nonincreasing absolute risk aversion. By enlarging membership, the firm simply spreads out the additional fixed costs over a greater number of partners.

Finally, let us examine the effect on employment and output of a change in the moments of the distribution of the random variable $u$. The partnership may revise its beliefs about the distribution of $u$ in light of new information about the cases at hand. The impact of a change in the expected value of $u$, with higher central moments constant, can be analyzed using a technique suggested by Sandmo [13] who replaces $u$ by $u^{\prime}=u+\theta$ in the first order condition and evaluates the effect of a change in $u$ at $\theta=o$ where $\theta$ is an additive shift parameter that moves the probability distribution to the right without affecting its shape. Differenciating implicitly equation (4) with respect to $\theta$ and to $N$ yields.

$$
d N / d \theta=-\left(1 / D_{1} N^{2}\right)\left\{b Q E U^{\prime \prime}(\lambda u+F / N)+\lambda N E U^{\prime}\right\}<0 .
$$

Under nonincreasing absolute risk aversion $d N / d \theta$ is negative. A change in the expected value of $u$ leads to an opposite change in employment and the volume of legal services. For instance, an increase in $u$ - meaning larger expected revenues - will cause the partnership to reduce the number of partners in the firm and decrease the volume of legal services. This perverse effect is somewhat similar to the case of the producer cooperative firm which has been shown to display a downward sloping supply curve. ${ }^{10}$ By reducing the existing level of

${ }^{9}$ It is assumed that the law firm can expand membership by accepting freely available new lawyers. Likewise it can contract membership by applying some method for selecting the leavers and/or by not replacing those lawyers who voluntarily leave the law firm.

${ }^{10}$ In this respect, see the works of Ward [16] and Domar [3]. 
employment in response to an increase in expected revenues, the nonincreasing absolute risk averse partnership can secure a larger income per member for those partners remaining with the firm.

The impact of a marginal increase in uncertainty on the equilibrium position of the partnership can be analyzed using again a technique proposed by Sandmo [13] who lets the distribution of $u$ undergo a mean preserving spread in which the distribution of $u$ is stretched out around its constant mean. A multiplicative shift parameter and an additive one $\theta$ are introduced and $u$ is replaced by $u^{\prime}=\gamma u+\theta$. In order to preserve the mean of the distribution, $\gamma$ and $\theta$ must have the following property

$$
d E(\gamma u+\theta)=0, \text { or } d \theta / d \gamma=-E u=-\bar{u} .
$$

Replacing $u$ by $u^{\prime}=\gamma u+\theta$ in equation (4), differentiating implicity with respect to $\gamma$ and to $N$, using condition (12) and evaluating the result at $\gamma=1, \theta=o$ yields equation (13) derived in the mathematical appendix

$$
\begin{aligned}
& d N / d \gamma=-\left(1 / D_{1} N^{2}\right)\left\{(b Q / \lambda)\left[E U^{\prime \prime}(\lambda+F / N)^{2}-(F / N+\lambda \bar{u}) E U^{\prime \prime}(\lambda u+F / N)\right]\right. \\
& \left.+\lambda N E U^{\prime}(u-\bar{u})\right\} .
\end{aligned}
$$

The sign of the last term is positive since $\lambda<0$ and $E U^{\prime}(u-\bar{u})=\operatorname{cov}\left(U^{\prime}, u\right)<0$. Under constant absolute risk aversion the first term in brackets is negative and $d N / d \gamma>0$. It is also positive under risk neutrality. Unfortunately, the sign of $d N / d \gamma$ is indeterminate for the decreasing absolute risk averse partnership. Thus constant absolute risk aversion is a sufficient condition for the risk averse partnership to increase the number of partners and the volume of legal services in response to a small increase in uncertainty. Note that this result is consistent with the previous conclusion showing that the risk averse partner employs and produces more under uncertainty.

\subsection{Clients' equilibrium position and the clients-lawyers' conflict}

Under ul.ct tainty, clients as a whole are assumed to maximize the expected utility of their share of the recovery or benefits awarded them. The objective of the clients is to maximize

$$
E U\left(Y_{c}\right)=E U(1-b) Q^{*}
$$

where $Q^{*}$ is a random variable. The optimal level of employment in the partnership and the volume of legal services that will maximize the clients' receipts are found at the point where

$$
\begin{aligned}
& E U^{\prime}\left(Y_{c}\right)(1-b) Q_{N}^{*}=0 \\
& \text { with } D_{c}=(1-b)\left(E U^{\prime \prime}\left(Y_{c}\right)\left(Q_{N}^{*}\right)^{2}+E U^{\prime}\left(Y_{c}\right) Q_{N N}^{*}<0 .\right.
\end{aligned}
$$

Equation (14) can be rewritten as

$$
E\left((1-b) Q_{N}^{*}=-(1-b) Q_{N} \operatorname{Cov}\left(U^{\prime}, u\right) / E U>0 .\right.
$$


Thus under risk aversion the expected value of the gross VMP of partners is positive at the point that maximizes the clients' receipts. Under certainty it is equal to zero and a conflict between the partnership and its clients may arise from the fact that their respective equilibrium positions do not coincide.

Referring to figure 1, observe that under certainty the equilibrium position desired by partners is at $N_{1}$ where VMP $=Y_{1}$ and the equilibrium position desired by the clients is at $N_{6}$ where VMP $=0$. Unless they interact, the partnership and its clients are faced with two conflicting equilibrium positions. The conflict area can be represented in figure 1 by $N_{1} A H$. Under uncertainty and risk aversion, the equilibrium position desired by the partnership is at $N_{2}$ where $E V M P<E Y_{1}$ and that desired by the clients is at $N$ where $E V M P>0$. A conflict is still possible but its resolution is more likely in this case since the conflict area is now smaller than under certainty. This is illustrated in figure l where the conflict area is equal to $N_{2} B G N_{5}$ under uncertainty and risk aversion. Note that for the profit-maximizing firm the conflict area is $\mathrm{N}_{4} \mathrm{FH}$ under certainty and $\mathrm{N}_{3} C G N_{5}$ under uncertainty and risk aversion. Thus a conflict is likely to be "smaller" under profit-maximizing behavior compared to the income-per-member-maximizing behavior, under both certainty as well as uncertainty and risk aversion.

Finally, consider the case where the risky output $Q^{*}$ is a function of both partners and non-partner personnel. The risk averse partnership maximizes $E U\left(Y_{2}\right)=E U\left(\left(b Q^{*}(N, n)-w n-\right.\right.$ $F) / N$ ) which yields the two first order equilibrium conditions

$$
\begin{aligned}
& E\left(b Q_{N}^{*}\right)=E Y_{2}-\lambda \operatorname{Cov}\left(U^{\prime}, u\right) / E U^{\prime}<E Y_{2} \\
& E\left(b Q_{n}^{*}\right)=w-b Q_{n} \operatorname{Cov}\left(U^{\prime}, u\right) / E U^{\prime}>w .
\end{aligned}
$$

Under certainty the covariances are zero and the expectations are equal to their argument. Thus the risk averse partnership employs more partners - as in the case where output was a function of $N$ only - but hires less non-partner personnel under uncertain CFC operations than it would under certainty. Note that the total output effect caused by uncertainty cannot be determined in this case since the two input effects have opposite signs.

The comparative-static equations for the two-input production function indicate that, under risk aversion, the effect of a change in share proportion $b$ on the employment of partners and non-partner personnel is indeterminate. All the other comparative-statics equations are also indeterminate in this case and, for the sake of compactness, are not presented in the paper.

\section{The model with contingency and noncontingency fee contracts}

\subsection{Firm's objective function and equilibrium position}

In this section the model is extended to incorporate CFC operations, the risky product, and NCFC operations, the riskless product, with both products assumed to be a function of two variable inputs, partners and non-partner personnel, and two fixed inputs, capital and the case loads as expressed in equation (1). It is further assumed that the proportion of the case loads between the two operations is fixed. In this case, the objective function of the firm is to maximize $Z_{3}$, the expected utility of the net-income-per-partner from both products, defined as

$$
Z_{3}=E U\left(Y_{3}\right)=E U\left[b_{1} Q_{1}^{*}\left(N_{1}, n_{1}\right)+b_{2} Q_{2}\left(N_{2}, n_{2}\right)-w n-F\right] / N
$$


where $w$ is the competitive wage rate of the non-partner personnel and $Q_{1}^{*}$ is the random output from CFC operations. The share proportions $b_{1}$ and $b_{2}$ are fixed parameters and the NCFC output $Q_{2}$ is not a random variable.

Given $N$, the total number of partners, and $n$, the total number of non-partner personnel, the firm must choose the number of partners $N_{1}$ and the number of non-partner personnel $n_{1}$ that should be allocated to the CFC operations. The remaining partners $N_{2}=N-N_{1}$ and non-partner personnel $n_{2}=n-n_{1}$ are assigned to the NCFC operations. In order to determine the optimal combination of $N_{1}$ and $n_{1}$, differentiate equation (18) with respect to $N_{1}$ and $n_{1}$, respectively, and set the derivatives equal to zero. This yields the two first order equilibrium conditions

$$
\begin{aligned}
& E\left(b_{1} Q_{1 N}^{*}\right)=b_{2} Q_{2 N^{-}} b_{1} Q_{1 N} \operatorname{Cov}\left(U^{\prime}, u\right) / E U^{\prime}>b_{2} Q_{2 N} \\
& E\left(b_{1} Q_{1 n}^{*}\right)=b_{2} Q_{2 n}-b_{1} Q_{1 n} \operatorname{Cov}(U, u) / E U^{\prime}>b_{2} Q_{2 n} .
\end{aligned}
$$

That is, in equilibrium, the risk averse partnership will allocate part ners and non-partner personnel so that the expected value of the net VMP for partners and non-partner personnel in the risky CFC operations exceed their respective net VMP in the riskless NCFC operations. Under certainty the covariance is zero and the net VMP of partners and non-partner personnel in the CFC operations are equal to their respective net VMP in the NCFC operations. Consequently, the response of the risk averse partnership to uncertain CFC operations is to shift partners and non-partner personnel from the now risky CFC activities to the riskless NCFC activities thus expanding the output of NCFC operations and simultaneously reducing the output of CFC operations. The profit-maximizing risk averse partnership will have the same response since the total employment of partners and non-partner personnel is fixed.

To determine the equilibrium level of total partner input, equation (18) is differentiated with respect to $N$, yielding"

$$
\begin{aligned}
& E\left(b_{1} Q_{1 N}^{*}\right)=E Y_{3}-b_{1}\left(Q_{1} / N_{1}\right)\left(e_{1}-\alpha\right) \operatorname{Cov}\left(U^{\prime}, u\right) / E U^{\prime} \\
& \text { where } e_{1}=Q_{1 N} /\left(Q_{1} / N_{1}\right) \text { and } \alpha=N_{1} / N \\
& \text { and } b_{2} Q_{2 N}=E Y_{3}-b_{1}\left(Q_{1} / N\right) \operatorname{Cov}\left(U^{\prime}, u\right) / E U^{\prime}>E Y_{3} .
\end{aligned}
$$

In equation (20-1), $e_{1}$ - the elasticity of $N_{1}$ in the production of $Q_{1}$ - is smaller than one under diminishing marginal products. The magnitude of the elasticity coefficient does not determine the sign of the second term in the RHS of equation (20-1) since $\alpha$ is smaller than one. Thus in the two-product case the expected value of the net VMP of total partner input in the production of the risky CFC operations may be smaller than, equal to, or larger than the expected value of the net income per partner at equilibrium, that is

$$
E\left(b_{1} Q_{1 N}^{*}\right) \gtreqless E Y_{3} .
$$

\footnotetext{
${ }^{u}$ Note that $\partial Z / \partial N=\left(\partial Z / \partial N_{1}\right)\left(\partial N_{1} / \partial N\right)=\left(\partial Z / \partial N_{1}\right)$

and $\partial Z / \partial N=\left(\partial Z / \partial N_{2}\right)\left(\partial N_{2} / \partial N\right)=\left(\partial Z / \partial N_{2}\right)$

Also $Q_{1 N}=Q_{1 N_{1}}$ and $Q_{2 N}=Q_{2 N_{2}}$.
} 
The partner-input effect and the output effect of uncertain CFC operations cannot be determined for the extended model.

From (20-2) it follows that, in equilibrium, the risk averse partnership will set the net VMP of total partner input in the production of the risless NCFC operations higher than the expected value of the net income per partner, thus reducing the employment of partners in the NCFC operations. However, the total employment of partners under uncertainty is determinate.

The equilibrium level of total non-partner input is determined by differentiating equation (18) with respect to $n$, obtaining

$$
\begin{aligned}
& E\left(b_{1} Q_{1 n}^{*}\right)=w-b_{1} Q_{1 n} \operatorname{Cov}\left(U^{\prime}, u\right) / E U^{\prime} \\
& b_{2} Q_{2 n}=w .
\end{aligned}
$$

From equations (22-1) and (22-2) it follows that in equilibrium and risk aversion.

$$
E\left(b_{1} Q_{1 n}^{*}\right)>b_{2} Q_{2 n}=w .
$$

The risk averse partnership achieves equilibrium at a level of total non-partner input for which the net VMP of non-partner personnel in the riskless NCFC operations equals their competitive wage and for which the expected value of the net VMP of non-partner personnel in the risky CFC operations exceeds their competitive wage. Under certainty the equilibrium level of total partner and non-partner inputs is achieved at the point where the net VMP of partners in both productions are equal to the net income per partner and the net VMP of nonpartner personnel in both productions are equal to their competitive wage. Thus, as in the case of a single product, the risk averse partnership will hire less of the non-partner personnel under uncertainty than it would under certainty.

\subsection{Comparative-static behavior}

The examination of the comparative-statics behavior of the partnership in the extended model will be restricted to its allocative equilibrium because of the existence of several indeterminacies in the comparative-statics equations for the total partner and non-partner equilibrium positions. ${ }^{12}$ In the case of the allocative equilibrium the partnership is on its production possibility curve producing the equilibrium combination of its risky and riskless output of legal services. By performing a comparative-statics analysis at the allocative equilibrium position it is possible to determine how the partnership will change the allocation of its fixed supply of partner and non-partner inputs between the two outputs in response to a change in one of the parameters of the model, namely, the share proportions $b_{1}$ and $b_{2}$, the wage $w$, the fixed costs $F$ and the moments of the distribution of $u$. The comparative-statics equations are derived in the mathematical appendix and the results are summarized in the last column of table 1 . Note that since $N$ and $n$ are fixed it follows that (i) the effect of a change in one of the

${ }^{12}$ The indeterminacies do not only arise from $\alpha$ different from one, but also from the presence of the term $\left(b_{2} Q_{2}-w L-F\right)$ in the comparative-statics equations. The sign of this term is clearly indeterminate. 


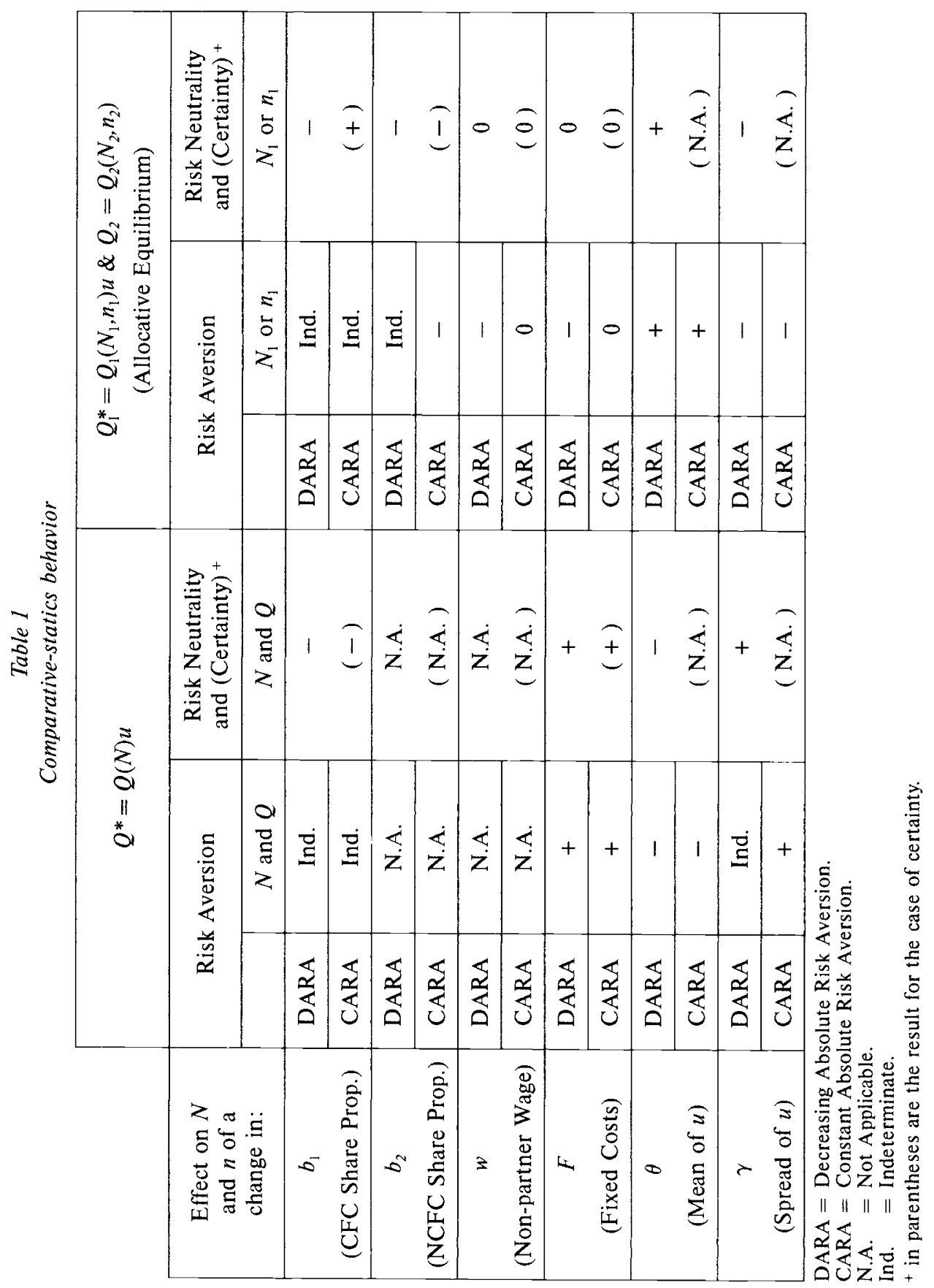


model's parameters on $N_{1}$ is the same as on $n_{1}$ and therefore any change in $N_{1}$ is accompanied by a change in $Q_{1}$ in the same direction and, as pointed out earlier, (ii) the conclusions drawn for the partnership which maximizes the net-income-per-partner hold true for the profit-maximizing partnership.

Referring to table 1 , observe that the effect of a change in the share proportion $b_{1}$ for the risky CFC operations is indeterminate under risk aversion and positive under risk neutrality and certainty. Thus assuming that shifts in inputs between the two productions are possiblea reasonable assumption for the partnership - the short run response of the risk neutral firm to an increase in $b_{1}$ will be to shift some of its inputs from the production of the risless output $Q_{2}$ to the production of the risky output $Q_{1}^{*}$ whose share proportion is now larger. The partnership will do the same under certainty but its behavior cannot be predicted under risk aversion regarless of the type of absolute risk aversion it displays.

The effect of a change in the share proportion $b_{2}$ for the riskless NCFC operations in only determined under constant absolute risk aversion (CARA), risk neutrality and certainty and its sign is negative. Thus if $b_{2}$ decreases the production of $Q_{2}$ will fall and that of $Q_{1}$ will rise under CARA, risk neutrality or certainty. The effect of a change in either the wage of the nonpartner personnel or the firm's fixed costs is negative under decreasing absolute risk aversion (DARA) and zero under CARA, risk neutrality and certainty. The DARA firm will shift its inputs toward the production of the riskless NCFC operations in response to an increase in either $w$ or $F$. Finally, the impact of a change in expected revenues (change in $\theta$ ) will lead to a change in $Q_{1}$ in the same direction if the partnership displays DARA, CARA or risk neutrality and a marginal increase in risk will cause the firm to shift its inputs toward the production of $Q_{2}$, the riskless output, under DARA, CARA or risk neutrality.

\section{Policy implications and concluding remarks}

The stochastic models examined in the previous sections clearly demonstrate that the equilibrium position and the comparative-statics behavior of the risk averse partnership are affected by the introduction of uncertain CFC operations. Some important policy implications can be drawn from the model under certainty and risk neutrality since in these cases the partnership will expand the employment of partners and the volume of legal services in response to a drop in share proportion. However, when uncertainty prevails it was shown that the response of the risk averse partnership to a change in share proportion is determinate. Consequently, if the "minimum fee schedule" currently recommended by bar associations is relaxed or eliminated then the possible reduction in share proportion that may result from increased competition in the legal service delivery market - assuming unrestricted entry of new firms - may not lead to an expansion in the employment of lawyers and the volume of legal services. It is thus not clear that policy makers can induce an expansion in the employment of lawyers and the volume of legal services through elimination or relaxation of the "minimum fee schedule" and the "no advertisement provision" recommended by bar associations and other professional associations.

It was also shown that the effect of a change in share proportion will lead to an opposite change in employment and output as the law firm becomes less risk averse. Somewhat informally, this result suggests that smaller law firms, which may be considered less risk averse than larger firms, are more likely to expand the employment of lawyers and the volume of 
legal services in response to a drop in share proportion, ceteris paribus. It is worth mentioning that any change in the share proportion will cause a redistribution of income between the law firm and its clients. This redistribution effect, however, cannot be easily evaluated since the impact of a drop in share proportion on total output is indeterminate under uncertainty.

The examination of the equilibrium position of the risk averse partnership under uncertain CFC operations has provided new insights concerning two issue : (1) the comparative size of the optimal input and output levels under per-partner-income-maximizing behavior versus profit-maximizing behavior and (2) the degree of conflict that may arise between the risk averse partnership and its clients. Under uncertain CFC operations, the input and output gaps between the risk averse per-partner-income-maximizing firm and the risk averse profit-maximizing firm are narrower than under certainty. Thus, in the presence of risky CFC operations the behavior of the two types of firms converges. As for the conflict between the risk averse partners and their risk averse clients, it was shown to be much smaller when risky CFC operations are taken into consideration. Thus a possible conflict is more likely to be resolved under uncertainty than in the case of certainty.

\section{Mathematical appendix}

\section{Derivation of Equation (9)}

The implicit differenciation of equation (4) with respect to $N$ and $b$ gives $d N / d b=-D_{1}^{-1} d^{2} Z_{1} / d b d N$

with $\quad d^{2} Z_{1} / d b d N=E\left(U^{\prime \prime}\left(d Y_{1} / d b\right)\left(d Y_{1} / d N\right)+U^{\prime \prime}\left(d^{2} Y_{1} / d b d N\right)\right)$

$$
\begin{aligned}
d Y_{1} / d b & =(Q / N) u \\
d Y_{1} / d N & =\left(b\left(Q_{N}-Q / N\right) u+F / N\right)(1 / N) \\
& =(b(Q / N)(e-1) u+F / N)(1 / N)=(\lambda u+F / N)(1 / N)
\end{aligned}
$$

$(d / d b)\left(d Y_{1} / d N\right)=(\lambda / b N) u$

thus $E U^{\prime \prime}\left(d Y_{1} / d b\right)\left(d Y_{1} / d N\right)=\left(Q / N^{2}\right) E U^{\prime \prime}(\lambda u+F / N) u$

but $\quad E U^{\prime \prime}(\lambda u+F / N) u=\lambda^{-1} E U^{\prime \prime}(\lambda u+F / N)(\lambda u+F / N-F / N)$

$$
=\lambda^{-1} E U^{\prime \prime}(\lambda u+F / N)^{2}-(F / \lambda N) E U^{\prime \prime}(\lambda u+F / N)
$$

hence $E U^{\prime \prime}\left(d Y_{1} / d b\right)\left(d Y_{1} / d N\right)=\left(Q / \lambda N^{2}\right)\left(E U^{\prime \prime}(\lambda u+F / N)^{2}-(F / N) E U^{\prime \prime}(\lambda u+F / N)\right)$

Also $E U^{\prime}\left(d^{2} Y_{1} / d b d N\right)=(\lambda / b N) E U^{\prime} u$

but $E U^{\prime}(\lambda u+F / N)=0$, the first order condition 
thus $\lambda E U^{\prime} u=-(F / N) E U^{\prime}$ and

hence $(\lambda / b N) E U^{\prime} u=-\left(F / N^{2} b\right) E U^{\prime}$

(A-1) and (A-2) yield equation (9).

\section{Derivation of equation (13)}

The implicit differenciation of equation (4) with respect to $\gamma$ and $N$ gives $d N / D \gamma=-D_{1}^{-1} E\left(U^{\prime \prime}\left(d Y_{1} / d \gamma\right)\left(d Y_{1} / d N\right)+U^{\prime}\left(d^{2} Y_{1} / d \gamma d N\right)\right)$

since $Y_{1}=(b Q(\gamma u+\theta)-F) / N$ and $d \theta / d \gamma=-\bar{u}$

then $\quad d Y_{1} / d \gamma=b Q(u-\bar{u}) / N$

and $(d / d \gamma)(d \gamma / d N)=\lambda(u-\bar{u}) / N$

thus $\quad d N / d \gamma=-D_{1}^{-1}\left(E U^{\prime \prime}(b Q(u-\bar{u}) / N)(\lambda u+F / N)+E U^{\prime} \lambda(u-\bar{u}) / N\right)$

$$
=-D_{1}^{-1}\left(\left(b Q / N^{2}\right) E U^{\prime \prime}(\lambda u+F / N)(u-\bar{u})+(\lambda / N) E U^{\prime}(u-\bar{u})\right)
$$

but $E U^{\prime \prime}(\lambda u+F / N)(u-\bar{u})=(1 / \lambda) E U^{\prime \prime}(\lambda u+F / N)(\lambda u+F / N-F / N-F / N-\lambda \bar{u})$

$$
=(1 / \lambda)\left(E U^{\prime \prime}(\lambda u+F / N)^{2}-(F / N+\lambda \bar{u}) E U^{\prime \prime}(\lambda u F / N)\right.
$$

which yields equation (13).

\section{Comparative-statics equation for the allocative equilibrium}

Introduce $v_{N}=b_{1}\left(Q_{1 N_{1}}\right) u-b_{2}\left(Q_{2 N_{2}}\right)$ and $v_{n}=b_{1}\left(Q_{1 n_{1}}\right) u-b_{2}\left(Q_{2 n_{2}}\right)$.

Then the first and second conditions for a maximum are

$\partial Z_{3} / \partial N_{1}=E U^{\prime} v_{N}=0$

$\partial Z_{3} / \partial_{n 1}=E U^{\prime} v_{N}=0$

$\partial^{2} Z_{3} / \partial N_{1}^{2}=A_{1}=E U^{\prime \prime} v_{N}^{2}+E U^{\prime}\left(b_{1} \partial Q_{1 N_{1}}^{*} / \partial N_{1}+b_{2} \partial Q_{2 N_{2}} / \partial N_{2}\right)<0$

$\partial^{2} Z_{3} / \partial n_{1}^{2}=A_{2}=E U^{\prime \prime} v_{n}^{2}+E U^{\prime}\left(b_{1} \partial Q_{1 n_{1}}^{*} / \partial n_{1}+b_{2} \partial Q_{2 n_{2}} / \partial n_{2}\right)<0$

and $A_{1} A_{2}-B_{1}^{2}=D_{3}>0$

where

$B_{1}=\partial^{2} Z_{3} / \partial N_{1} \partial n_{1}=E U^{\prime \prime} v_{N} v_{n}+E U^{\prime}\left(b_{1} \partial Q_{N_{1}}^{*} / \partial n_{1}+b_{2} \partial Q_{2 N_{2}} / \partial n_{2}\right.$. 
Using (A.3.1), (A.3.2) and the expression for $v_{N}$ and $v_{n}$ it can be easily shown that

$$
\begin{array}{ll} 
& Q_{1 N_{1}} / Q_{1 n_{1}}=Q_{2 N_{2}} / Q_{2 n_{2}} \\
\text { and } & v_{N}=\left[Q_{1 N_{1}} / Q_{1 n_{1}}\right] \cdot v_{n} \\
\text { or } & v_{N}=a \cdot v_{n} .
\end{array}
$$

Suppose $p_{i}$ is the model's parameter with respect to which comparative-statics is performed where $p_{i}$ is equal to either one of the following parameters: $b_{1}, b_{2}, w, F, \theta$, or $\gamma$. Differenciate (A.3.1) and (A.3.2) with respect to $p_{i}$ yields, respectively

$$
\begin{aligned}
& A_{1} \frac{\partial N_{1}}{\partial p_{i}}+B_{1} \frac{\partial n_{1}}{\partial p_{i}}=-\frac{\partial}{\partial p_{i}}\left(\frac{\partial Z_{3}}{\partial N_{1}}\right) \equiv G_{p_{i}}^{1} \\
& B_{1} \frac{\partial N_{1}}{\partial p_{i}}+A_{2} \frac{\partial n_{1}}{\partial p_{i}}=-\frac{\partial}{\partial p_{i}}\left(\frac{\partial Z_{3}}{\partial n_{1}}\right) \equiv G_{p_{i}}^{2}
\end{aligned}
$$

solving the preceding system gives

$$
\frac{\partial N_{1}}{\partial p_{i}}=\frac{G_{p i}^{1} A_{2}-G_{p_{i}}^{2} B_{1}}{D_{3}} \quad \frac{\partial n_{1}}{\partial p_{i}}=\frac{G_{p i}^{2} A_{1}-G_{p_{i}}^{1} B_{1}}{D_{3}}
$$

It can be shown that for all $p_{i}$ the following relationship is always satisfied

$$
G_{p_{i}}^{1}=a \cdot G_{p_{i}}^{2}
$$

where $\mathrm{a}$ is defined in (A.3.8) and (A.3.8'). Consequently, (A.3.11) can be rewritten as

$$
\frac{\partial N_{1}}{\partial p_{i}}=\frac{G_{p_{i}}^{2}\left(a A_{2}-B_{1}\right)}{D_{3}} \quad \frac{\partial n_{1}}{\partial p_{i}}=\frac{G_{p_{i}}^{2}\left(A_{1}-a B_{1}\right)}{D_{3}} .
$$

Since $D_{3}$ is positive, the signs of $\partial N_{1} / \partial p_{i}$ and $\partial n_{1} / \partial p_{i}$ are determined by that of $G_{p_{i}}^{2},\left(a A_{2}-B_{1}\right)$ and $\left(A_{1}-a B_{1}\right)$. From (A.3.3), (A.3.4) and (A.3.6) it follows that

$$
\begin{aligned}
& a A_{2}-B_{1}=a E U^{\prime}\left(b_{1} \partial Q_{1 n_{1}}^{*} / \partial n_{1}+b_{2} \partial Q_{2 n_{2}} / \partial n_{2}\right)-E U^{\prime}\left(b_{1} \partial Q_{1 N_{1}}^{*} / \partial n_{1}+b_{2} \partial Q_{2 N_{2}} / \partial n_{2}\right) \\
& A_{1}-a B_{1}=E U^{\prime}\left(b_{1} \partial Q_{1 N_{1}}^{*} / \partial N_{1}+b_{2} \partial Q_{2 N_{2}} / \partial N_{2}\right)-a E U^{\prime}\left(b_{1} \partial Q_{1 N_{1}}^{*} / \partial n_{1}+b_{2} \partial Q_{2 N_{2}} / \partial n_{2}\right)
\end{aligned}
$$

Given well-behaved production functions, that is $\partial Q_{N} / \partial n<0, \partial Q_{n} / \partial n<0$ and $\partial Q_{N} / \partial n>0$ then $\left(a A_{2}-B_{1}\right)$ and $\left(A_{1}-a B_{1}\right)$ are negative. Thus the signs of $\partial N_{1} / \partial p_{i}$ and $\partial n_{1} / \partial p_{i}$ are identical and equal to the opposite of that of $G_{p_{i}}^{2}$ 
The expressions for $G_{p_{i}}^{2}$ where $p_{i}=b_{1}, b_{2}, w, F, \theta$, and $\gamma$ are

3.1. Effect of a change in $b_{1}$ :

$$
G_{b_{i}}^{2}=-\left\{Q_{1}\left(N b_{1} Q_{1 n_{1}}\right)^{-1}\left[E U^{\prime \prime} v_{n}^{2}+b_{2} Q_{2 n_{2}} E U^{\prime \prime} v_{n}\right]+Q_{1 n_{1}} E U^{\prime} u\right\}
$$

3.2. Effect of a change in $b_{2}$ :

$$
G_{b_{2}}^{2}=-\left\{Q_{2} N^{-1} E U^{\prime \prime} v_{n}-Q_{2 n_{2}} E U^{\prime} u\right\}
$$

3.3. Effect of a change in $w$ :

$$
G_{w}^{2}=n N^{-1} E U^{\prime \prime} v_{n}
$$

3.4. Effect of a change in $F$ :

$$
G_{F}^{2}=N^{-1} E U^{\prime \prime} v_{n}
$$

3.5. Effect of a change in $\theta$ :

$$
G_{\theta}^{2}=-b_{1} N^{-1}\left\{Q_{1} E U^{\prime \prime} v_{n}+Q_{1 n 1} E U^{\prime \prime}\right\}
$$

3.6. Effect of a change in $\gamma$ :

$$
G_{\gamma}^{2}=-\left\{Q_{1}\left(N Q_{1 n 1}\right)^{-1}\left[E U^{\prime \prime} v_{n}^{2}-k_{n} E U^{\prime \prime} v_{n}\right]+b_{1} Q_{1 n 1} E U^{\prime}(u-\bar{u})\right\}
$$

where $k_{n}=\left(b_{1} Q_{1 n_{1}}\right) \bar{u}-\left(b_{2} Q_{2 n_{2}}\right)>0$ as indicated by equation 19-2.

To determine the sign of $G_{p_{i}}^{2}$ when possible, note that $E U^{\prime \prime} v_{n} \gtreqless 0$ according as absolute risk aversion decreases, remains constant, or increases (for a proof, see the information in footnote 7). Also, $E U^{\prime \prime} v_{n}^{2}<0, E U^{\prime} u>0, E U^{\prime}(u-\bar{u})<0$ and $E U^{\prime}>0$ under risk aversion.

\section{REFERENCES}

1. ARROW, K.J.: Essays in the Theory of Risk Bearing, North Holland Publishing Company, Amsterdam, 1974, Chapter 3.

2. BARON, D.P.: "Price uncertainty, utility, and industry equilibrium in pure competition", International Economic Review (October 1970), 463-480. 
3. DOMAR, E.D.: "The soviet collective farms as a producer cooperative", American Economic Review (September 1966), 734-757.

4. HAWAWINI, G. and MICHEL, P.: "Theory of the risk averse producer cooperative firm facing uncertain demand", Annals of Public and Cooperative Economy (April/June 1979), 43-62.

5. HAWAWINI, G.: "Multiple variable factors, demand uncertainty and the cooperative firm", Economics Letters 5 (1980), 15-19.

6. HAWAWINI, G.: "Uncertainty and the production decisions of owner-managed and labourmanaged firms", Oxford Economic Papers (March 1984), 119-130.

7. KIHLSTROM, R., ROMER, D. and WILLIAMS, S. : "Risk aversion with random initial wealth", Econometrica (July 1981), 911-920.

8. KWON, J.K.: "A model of the law firm", Southern Economic Journal (September 1978), 63-74.

9. MEADE, J.E.: "The theory of labour-managed firms and of profit sharing", Economic Journal (March 1972 (Supplement)), 402-427.

10. OI, W. and CLAYTON, E.: "A peasant's view of a soviet collective farm", American Economic Review (March 1968), 37-59.

11. PAROUSH, J. and KAHANA, N.: "Price uncertainty and the cooperative firm", American Economic Review (Mach 1980), 65-73.

12. PRATT, J.W.: "Risk aversion in the small and in the large", Econometrica (January 1964), 122-136.

13. SANDMO, A.: "On the theory of the competitive under price uncertainty", American Economic Review (March 1971), 65-73.

14. VANEK, J.: The General Theory of Labour-Managed Economies, Cornell University Press, Ithaca, 1970.

15. VON NEUMANN, J. and MORGENSTERN, O.: Theory of Games and Economic Behavior, Third Edition, Princeton University Press, Princeton, 1953.

16. WARD, B.: "The firm in Illyria : market syndicalism", American Economic Review (September 1958), 566-589. 